\title{
A Scalable Three-Step Approach for Demand Side Management of Plug-in Hybrid Vehicles
}

\author{
Stijn Vandael, Member, IEEE, Bert Claessens, Member, IEEE, Maarten Hommelberg, Member, IEEE, \\ Tom Holvoet, Member, IEEE, and Geert Deconinck, Senior Member, IEEE
}

\begin{abstract}
In this paper, we present a scalable approach for DSM (demand side management) of PHEVs (plug-in hybrid electric vehicles). Essentially, our approach consists of three steps: aggregation, optimization, and control. In the aggregation step, individual PHEV charging constraints are aggregated upwards in a tree structure. In the optimization step, the aggregated constraints are used for scalable computation of a collective charging plan, which minimizes costs for electricity supply. In the real-time control step, this charging plan is used to create an incentive signal for all PHEVs, determined by a market-based priority scheme. These three steps are executed iteratively to cope with uncertainty and dynamism. In simulation experiments, the proposed three-step approach is benchmarked against classic, fully centralized approaches. Results show that our approach is able to charge PHEVs with comparable quality to optimal, centrally computed charging plans, while significantly improving scalability.
\end{abstract}

Index Terms-Demand side management, market-based control, plug-in hybrid electric vehicles.

\section{INTRODUCTION}

G LOBAL environmental concerns, decreasing fossil fuel reserves, and government policies are expected to increase the share of renewables in global electricity generation from $19 \%$ in 2008 to almost a third in 2035 [1]. The intermittent nature of this type of electricity generation, combined with the rising electricity demand, requires an efficient usage of the available electricity generation. One solution for this problem is DSM (demand side management).

DSM is the modification of user demand for optimal usage of the electrical grid and its connected generation units. Currently, utilities use two techniques to achieve DSM: direct load control (DLC) and real-time pricing. Direct load control is a DSM technique where large consumers allow utilities to control part of their electricity demand. An example application of direct load control is the control of dimmers within a building's lighting system [2]. Real-time pricing is a DSM technique where consumers pay a variable tariff, which reflects the utility's cost of generating or purchasing electricity at a wholesale level. An example of real-time pricing is the usage of smart meters in California, which vary prices by season and time of day [3].

Manuscript received October 09, 2011; revised March 04, 2012, June 15, 2012, July 24, 2012, July 31, 2012; accepted August 01, 2012. The work of $\mathrm{S}$. Vandael is supported by the Institute for the Promotion of Innovation by Science and Technology in Flanders (IWT). Paper no. TSG-00573-2011.

S. Vandael and T. Holvoet are with the Department of Computer Science, KU Leuven, 3001 Leuven, Belgium.

B. Claessens and M. Hommelberg are with the Energy Department of the Research Institute VITO, $2400 \mathrm{Mol}$, Belgium.

G. Deconinck is with the Department of Electrical Engineering, KU Leuven, 3001 Leuven, Belgium.

Digital Object Identifier 10.1109/TSG.2012.2213847
Current research concerning DSM is focussed on large-scale DSM of local devices, such as domestic appliances and electric cars [4], [5].

A PHEV is a car with both an electric motor and a combustion engine, together with a battery that can be charged through a plug. Coordinated charging of electric vehicles in a smart grid is an important research topic, because DSM of PHEVs offers an opportunity for managing fluctuations in electricity generation and consumption [6]. To enable DSM of PHEVs, two major challenges were identified.

The first challenge is the constantly increasing number of PHEVs. By 2035, plug-in hybrid vehicles and electric vehicles are expected to constitute around $40 \%$ of the global car sales [1]. Consequently, a scalable DSM approach for PHEVs is necessary.

The second challenge is the uncertainty and dynamism inherent to DSM of PHEVs. For example, PHEVs are continuously connected and disconnected from the grid at uncertain times. Consequently, a DSM approach has to take into account dynamic user behavior.

Driven by these challenges, we present an approach for DSM of PHEVs. The main contributions of this paper are:

- Description of a three-step approach, which achieves scalable computation of a charging plan, based on aggregated PHEV charging constraints. The short execution times enable fast, continuous iterations of the three steps, in order to cope with dynamism and uncertainty.

- Evaluation of the three-step approach through benchmarking against two fully-centralized approaches, defined by using QP (Quadratic Programming). This evaluation shows that our approach significantly improves scalability for an increasing number of PHEVs and optimization horizon.

\section{RELATED WORK}

In research studies concerning smart grids, several approaches are proposed for DSM of local devices. These approaches can be classified in two categories: centralized and decentralized (agent-based) approaches. In centralized approaches, a central optimizer has all information about the devices in its control area. In agent-based approaches, devices locally optimize their energy usage, while externally exchanging a limited amount of information. This section gives a representative selection of centralized and decentralized approaches in smart grids and the position of our three-step approach in current research. 


\section{A. Centralized Approaches for Smart Grids}

In centralized approaches for smart grids, energy usage of individual devices is centrally scheduled. Central scheduling is typically achieved by state of the art scheduling techniques, including linear programming, quadratic programming, (approximate) dynamic programming, and stochastic programming [7], [8].

Centralized approaches for DSM of PHEVs are proposed in [9], for minimizing power losses in distribution grids, and in [6], for correcting errors in wind predictions. Small-scale evaluations show that these approaches are able to find the best possible solution, based on the available data. In terms of scalability, Galus et al. [6] report that for a large number of PHEVs, memory constraints are violated.

Centralized approaches based on approximate dynamic programming (ADP) are proposed in [10], for management of distributed generation and storage, and in [11], for a V2G aggregator who optimizes bidirectional power flows of PHEVs. The results of these experiments show that the ADP solutions are suboptimal, while limiting computation times.

In summary, centralized approaches can guarantee optimal solutions, given the available data by using mathematical optimization techniques. While approximate methods can reduce execution times, computational complexity is usually unavoidable due to the large amount of data about individual devices. In agent-based research, centralized approaches can be used as a benchmark.

\section{B. Decentralized Approaches for Smart Grids}

In decentralized (agent-based) approaches for smart grids, devices make autonomous decisions (through a software agent) concerning their energy usage.

In [4], an iterative, agent-based control concept is presented. In this approach, all devices individually determine their energy schedule, which is aggregated in a hierarchical structure. Based on the aggregated energy schedule, a global controller iteratively determines an appropriate incentive signal to reach a global objective. Results show that good local predictions lead to a better matching of demand and supply.

In [5], the authors propose a consumption scheduling game. This game provides the proof that the energy management approach converges, finds the optimal solution, and avoids users from cheating.

In summary, agent-based approaches can provide a solution which naturally maps onto a distributed environment. A recurring strategy in current approaches is computing a local schedule and adjusting this schedule until a global equilibrium is reached. An inherent challenge in these strategies is controlling the emergent consumption schedule for a group of devices.

\section{Position of Our Three-Step Approach}

Our approach is not fully centralized, neither fully decentralized. While the charging plan for a PHEV fleet (group of PHEVs contracted by the same energy supplier) is calculated centrally, the control power of individual PHEVs is calculated locally (Table I).

The most significant difference between our approach and centralized approaches is that the central part of our approach
TABLE I

COMPARISON OF DSM APPROACHES IN TERMS OF DECISION MAKING AT DifFerent LeVEls $(\mathrm{L}=$ LOCAL, $\mathrm{C}=$ CENTRAL)

\begin{tabular}{|l||c|c|c|}
\hline DSM decisions & $\begin{array}{c}\text { A. Centralized } \\
\text { approaches }\end{array}$ & $\begin{array}{c}\text { B. Decentralized } \\
\text { approaches }\end{array}$ & $\begin{array}{c}\text { C. Three-step } \\
\text { approach }\end{array}$ \\
\hline \hline energy planning & $\mathrm{C}$ & $\mathrm{L}$ & $\mathrm{C}$ \\
\hline power control & $\mathrm{C}$ & $\mathrm{L}$ & $\mathrm{L}$ \\
\hline
\end{tabular}

(step 2) does not calculate an individual charging plan for each PHEV. Rather, a collective charging plan for all PHEVs is calculated, which is less computationally intensive. In step 3, this collective charging plan is translated to individual PHEV power setpoints through a market-based priority scheme.

The difference between our approach and decentralized approaches, is that the collective PHEV consumption is not a result of iterations of locally proposed charging plans. Instead, the collective PHEV consumption is a result of a centrally calculated charging plan (step 2). For energy suppliers, central availability of a charging plan is important for assessing the costs associated with supplying energy to their PHEV fleet.

\section{BACKGROUND: MARKET-BASED CONTROL}

The DSM approach presented in this paper uses demand functions from market-based MAS (multi-agent system) control [12]. In market-based MAS control, demand and supply functions are used to match demand and supply in a cluster of devices (Fig. 1). A demand function represents the prices a consumer is willing to pay for different power consumption values, while a supply function represents the prices a producer is willing to accept for different power generation values.

The matching process in market-based control is coordinated by an auctioneer agent. First, the auctioneer agent gathers demand and supply functions from all device agents in the cluster. These device agents control a local device (e.g., PHEV, diesel generator). Then, the auctioneer agent finds an equilibrium price, at which device agents are willing to consume and generate the same amount of power. Finally, the equilibrium price is announced to all device agents in the cluster and device agents will start consuming and producing appropriately. The fundamentals of this mechanism originate from traditional demand and supply matching in economics.

While market-based control is a well-known approach for matching demand and supply, many challenges remain to be solved. One of these challenges is ahead-planning of energy usage in an uncertain and dynamic environment.

\section{ThreE-STEP APPROACH FOR DSM OF PHEVS}

Based on the identified challenges (Section I), a novel threestep, agent-based approach for DSM of PHEVs is presented. In this approach, a PHEV agent represents its PHEV at the local domestic level, while a PHEV fleet agent represents the energy supplier, which globally manages a number of contracted PHEVs (called the energy supplier's "PHEV fleet"). Agents have the following goals:

- PHEV agent

Charge PHEV battery before departure time, while respecting local power limitations (e.g., maximum power of a household connection). 


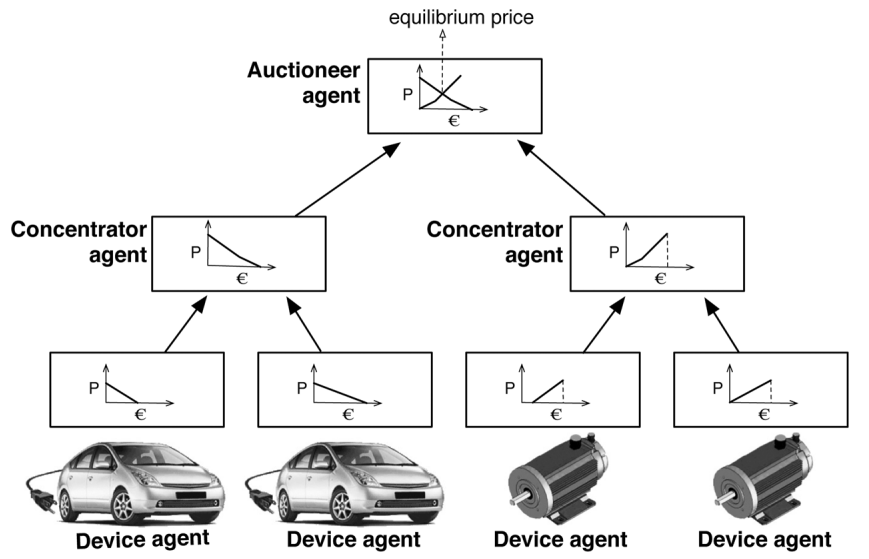

Fig. 1. Market-based control.

\section{- PHEV Fleet agent}

Charge PHEV fleet to minimize costs for energy supply (dependent on production units managed by the energy supplier, prices at the wholesale electricity market, etc.).

To take into account all individual PHEV constraints, while minimizing energy supply costs, the agents coordinate in three steps (Fig. 2). In the first step, local PHEV constraints are aggregated towards the PHEV fleet agent. In the second step, the aggregated constraints are used for scalable computation of a collective charging plan for the PHEV fleet, which minimizes energy supply costs. In the third step, a market-based control signal is propagated from the fleet agent towards the PHEV agents. While computational scalability is achieved through aggregation of constraints (step 2), adaptability to a dynamic, uncertain environment is achieved through continuous iterations of the three steps.

\section{A. Step 1: PHEV Constraints Aggregation}

The goal of this step is to inform the fleet agent about the collective charging constraints of its PHEV fleet. The charging constraints of a PHEV can be classified in two types of constraints: energy constraints and power constraints. Energy constraints express the limits to which energy can be shifted in time, while power constraints express the limits to which the momentary charging power of PHEVs can be adjusted. Both types of constraints can be aggregated to represent collective energy and power constraints of a PHEV fleet.

Throughout the paper, $\mathcal{F}$ is defined as the set of PHEVs contained within the PHEV fleet, and $\mathcal{F}_{c, t} \subset \mathcal{F}$ is defined as the subset of PHEVs which are grid-connected at time $t$. For each PHEV $i \in \mathcal{F}_{c, t},{ }^{i} P_{t}$ is defined as the power drawn at time $t$, and ${ }^{i} E_{t}$ as the energy charged at time $t$. Granularity of time is determined by the time interval $\Delta t$, chosen between two iterations of the three-step approach. This choice is based on the business model used by the energy supplier. For example, if energy is bought at a wholesale market, $\Delta t$ equals the length of a trading period. Consequently, we assume PHEV charging plans are discretized according to these trading periods.

The individual energy constraints of a PHEV $i$ at time $t=0$ can be specified by its required energy at departure time ${ }^{i} t_{\text {dep }}$ :

$$
{ }^{i} E_{t}=0 \quad t \in\{0\}
$$

STEP 1

constraints aggregation

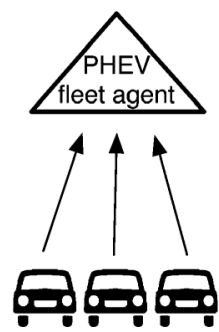

STEP 2

fleet optimization
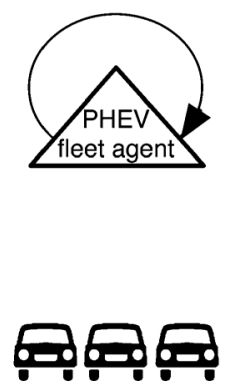

STEP 3

real-time control

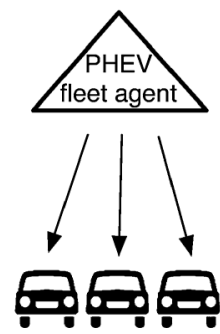

Fig. 2. Three-step approach.

$$
\begin{array}{lrl}
{ }^{i} E_{t}={ }^{i} E^{\mathrm{req}} & t \in & \left\{{ }^{i} t_{\mathrm{dep}}\right\} \\
{ }^{i} E_{t+1}={ }^{i} E_{t}+{ }^{i} P_{t} \Delta t & \forall t & \in\left\{0, \ldots,{ }^{i} t_{\mathrm{dep}}\right\} \\
0 \leq{ }^{i} P_{t} \leq{ }^{i} P^{\max } & \forall t & \in\left\{0, \ldots,{ }^{i} t_{\mathrm{dep}}\right\} \\
\forall i \in \mathcal{F}_{c, 0} &
\end{array}
$$

where:

- ${ }^{i} E^{\text {req }}$ is the energy that a PHEV $i$ needs to charge between the current time 0 and its departure time ${ }^{i} t_{\mathrm{dep}}$.

- ${ }^{i} P^{\max }$ is the maximum power at which a PHEV $i$ can charge its battery. ${ }^{i} P^{\max }$ can be dependent on the power limitations of the battery, the BMS (battery management system) or the local grid connection. E.g., at a fast charging station, PHEVs can charge at a higher power than at a regular household connection.

Note that time $t$ is expressed relative, where $t=0$ is defined as the time where step 1 is initiated.

Constraints (1)-(4) are translated to the energy constraint vectors ${ }^{i} \mathbf{E}^{\max }$ and ${ }^{i} \mathbf{E}^{\text {min }}$ for a each PHEV $i$ at time $t=0$, which will be used in the calculation of the PHEV fleet charging plan (step 2, Section IV-B):

$$
\begin{gathered}
{ }^{i} \mathbf{E}^{\max }=\left\{{ }^{i} E_{t}^{\max } \mid{ }^{i} E_{t}^{\max }=\min \left({ }^{i} P^{\mathrm{max}} t,{ }^{i} E^{\mathrm{req}}\right),\right. \\
\left.\forall t \in\left\{0, \ldots,{ }^{i} t_{\mathrm{dep}}\right\}\right\} \\
{ }^{i} \mathbf{E}^{\mathrm{min}}=\left\{{ }^{i} E_{t}^{\mathrm{min}} \mid{ }^{i} E_{t}^{\mathrm{min}}=\max \left({ }^{i} E^{\mathrm{req}}-{ }^{i} P^{\max }\left({ }^{\mathrm{min}}{ }_{\mathrm{dep}}-t\right), 0\right),\right. \\
\left.\forall t \in\left\{0, \ldots,{ }^{i} t_{\mathrm{dep}}\right\}\right\} \\
\forall i \in \mathcal{F}_{c, 0}
\end{gathered}
$$

An example of these battery constraints is shown in Fig. 3(a). The area between ${ }^{i} \mathbf{E}^{\max }$ and ${ }^{i} \mathbf{E}^{\text {min }}$ contains all possible charging values ${ }^{i} E_{t}$.

The individual power constraints of a PHEV $i$ at time $t=0$ are represented in a demand vector ${ }^{i} \mathbf{P}^{\mathrm{dem}}$, which contains all possible power values for charging the PHEV's battery. These power values vary between ${ }^{i} P^{\min }$ and ${ }^{i} P^{\max }$ [Fig. 4(a)], and are specified by a self-defined piecewise linear function ${ }^{i} f_{d}$.

$$
\begin{aligned}
& { }^{i} f_{d}\left(p^{r}\right)=\left\{\begin{array}{lc}
{ }^{i} P^{\max }-p^{r}\left(\frac{{ }^{i} P^{\max }-{ }^{i} P^{\min }}{{ }^{i} p_{c}^{r}}\right): & 0 \leq p^{r}<{ }^{i} p_{c}^{r} \\
{ }^{i} P^{\min } & :{ }^{i} p_{c}^{r} \leq p^{r} \leq p_{\text {max }}^{r}
\end{array}\right. \\
& { }^{i} \mathbf{P}^{\text {dem }}=\left\{{ }^{i} P_{p^{r}}^{\text {dem }} \mid{ }^{i} P_{p^{r}}^{\text {dem }}=f_{d}\left(p^{r}\right), \forall p^{r} \in\left\{0, \ldots, p_{\max }^{r}\right\}\right\}
\end{aligned}
$$




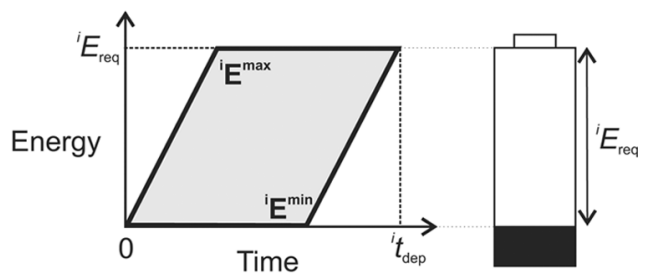

(a)

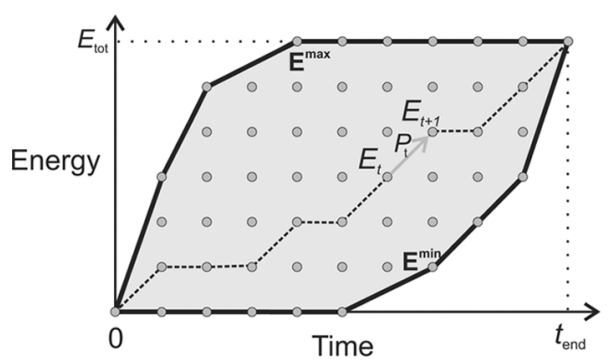

(b)

Fig. 3. PHEV energy constraints. (a) Individual energy constraints. (b) Aggregated energy constraints.

with ${ }^{i} p_{c}^{r}$ the corner priority of PHEV $i$ (defined in step 3 ). The priority domain $p^{r}$ of a demand function represents the charging priority, which indicates the wish (priority) for consuming or producing at a certain power. This charging priority is a more general representation of consumer incentives, compared to specific prices in market-based control. In Section IV-C (step 3, "real-time control"), the corner priority is explained in more detail.

Once the PHEV agents have assembled their individual energy and power constraint vectors, as defined in (5), (6), and (8), the vectors are aggregated:

$$
\begin{aligned}
& \mathbf{E}^{\max }=\sum_{i=1}^{n}{ }^{i} \mathbf{E}^{\max } \\
& \mathbf{E}^{\min }=\sum_{i=1}^{n}{ }^{i} \mathbf{E}^{\min } \\
& \mathbf{P}^{\mathrm{dem}}=\sum_{i=1}^{n}{ }^{i} \mathbf{P}^{\mathrm{dem}}
\end{aligned}
$$

where $n=\left|\mathcal{F}_{c, 0}\right|$, the amount of grid-connected PHEVs. An example of aggregated energy constraints is shown in Fig. 3(b), and an example of aggregated power constraints in Fig. 4(b).

The final result of the first step in our approach is the aggregated constraints of the PHEV fleet, located at the PHEV fleet agent. In step 2, the aggregated energy constraint vectors are used in the calculation of a collective charging plan for the PHEV fleet, and in step 3, the aggregated power constraint vector is used to control individual PHEVs.

\section{B. Step 2: Optimization of the PHEV Fleet}

The goal of this step is to determine a collective charging plan for the PHEV fleet, which minimizes costs for the energy supplier. The cost model for charging a PHEV fleet is dependent on the considered business model of an energy supplier. Among others, this cost model can contain distribution costs, generation costs, and energy bought or sold at energy markets (e.g., at

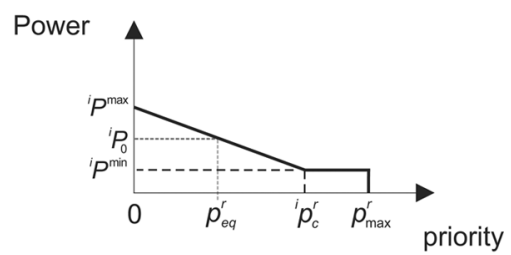

(a)

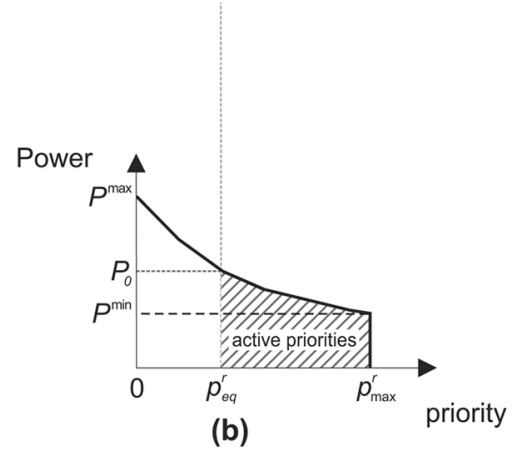

Fig. 4. PHEV power constraints. (a) Individual power constraints. (b) Aggregated power constraints.

the day-ahead market). In general, these costs can be defined by an objective function $\mathrm{C}$, which needs to be minimized. PHEV charging requirements are taken into account through the aggregated energy constraints (9) and (10). Both cost function and aggregated constraints are used to define the complete optimization problem for PHEV fleet charging:

$$
\min _{\mathbf{P}} C(\mathbf{P})
$$

subject to:

$$
\begin{aligned}
0 & \leq P_{t} \leq P^{\text {limit }} & & \forall t \in\left\{0, \ldots, t_{\text {end }}\right\}, \\
E_{t}^{\min } & \leq E_{t} \leq E_{t}^{\max } & & \forall t \in\left\{0, \ldots, t_{\text {end }}\right\}, \\
E_{t+1} & =E_{t}+P_{t} \Delta t & & \forall t \in\left\{0, \ldots, t_{\text {end }}\right\} .
\end{aligned}
$$

where $t_{\text {end }}$ is the latest departure time among all vehicles, and

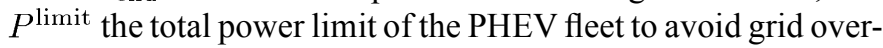
load. The result of solving the PHEV fleet charging problem is the control vector $\mathbf{P}$, which defines control values for the PHEV fleet.

Dependent on the shape of the objective function $\mathrm{C}$, several solution methods are applicable (e.g., linear programming, quadratic programming). In this paper, we have chosen to use dynamic programming (DP) [8] as generic solution method to allow inclusion of a diverse range of cost functions. To enable usage of DP, the optimization problem is redefined as a dynamic decision problem [13]. In general, a dynamic decision problem consists of a state space $\mathcal{S}$ and decision space $\mathcal{D}$. For the PHEV fleet charging problem, the state space is defined by a number of energy states $E_{t} \in \mathcal{S}$, which represent an amount of energy off-taken by the PHEV cluster after time $t$. The state space is constraint by $\mathbf{E}^{\max }$ and $\mathbf{E}^{\min }$, as can be seen in (14). A visual representation of all states $E_{t}$ in the state space are shown in Fig. 3(b). The decision space is defined by a number of charging decisions $P_{t} \in \mathcal{D}$. At each energy state $E_{t}$, a decision $P_{t}$ translates $E_{t}$ to a new energy state $E_{t+1}$ as in (15). The decision space is constraint by $P^{\text {limit }}$ as in (13). A visual representation of an action $P_{t}$ in the action space is shown in Fig. 3(b). 
The objective function in (12) is redefined into a recursive value function, known as the Bellman equation [8]:

$$
V\left(E_{t}\right)=\min _{a_{t}}\left\{F\left(E_{t}, a_{t}\right)+V\left(E_{t+1}\right)\right\}
$$

where $\mathrm{F}$ is the price of action $a_{t}$ in state $E_{t}$. By applying backwards induction to the Bellman equation, the PHEV fleet problem is solved. The result of solving this equation is a sequence of decisions which minimizes the objective function C. In terms of our problem, where a decision is a power value, this sequence of decisions is a charging plan $\mathbf{P}_{\text {ctrl }}$ for the entire fleet:

$$
\mathbf{P}_{\text {ctrl }}=\left\{P_{0}, P_{1}, P_{2}, \ldots, P_{t_{\text {end }}-1}\right\}
$$

The first decision $P_{0}$ is used to control the PHEVs in realtime, by translating $P_{0}$ to individual PHEV charging power values (step 3).

\section{Step 3: Real-Time Control}

The objective of this step is to divide $P_{0}$ in (17) between the individual PHEVs of the fleet. The deployed division strategy is based on the PHEV demand vector in (8). For every priority, this demand vector represents a certain charging power, where charging power slopes down from low to high priorities. This shape is similar to a classic demand function in economics, where demand slopes down from low to high prices.

The corner priority ${ }^{i} p_{c}^{r}$ [Fig. 4(a)] is a heuristic which determines the slope of a demand function. Increasing values of ${ }^{i} p_{c}^{r}$ decrease the slope, which indicates that PHEV $i$ wants to charge more at an increasing amount of priority values. Consequently, ${ }^{i} p_{c}^{r}$ is based on the "urgency" for PHEV $i$ to charge its battery. The formula of ${ }^{i} p_{c}^{r}$ is based on a heuristic used in [6]:

$$
{ }^{i} p_{c}^{r}=\frac{{ }^{i} E^{\mathrm{req}}}{{ }^{i} t_{\mathrm{dep}}{ }^{i \max }} \quad \forall i \in \mathcal{F}_{c, 0}
$$

Note that these three parameters also define the individual PHEV constraints (1)-(4).

The aggregated demand vector in (11) is used to translate the first decision $P_{0}$ of the charging plan to the equilibrium priority $p_{\text {eq }}^{r}$ [Fig. 4(b)]:

$$
p_{\text {eq }}^{r}=\underset{p^{r}=0, \ldots, p_{\max }^{r}}{\arg \min }\left|\mathbf{P}_{p^{r}}^{\text {dem }}-P_{0}\right|
$$

Subsequently, this equilibrium priority is sent as an incentive signal to all PHEV agents. Upon receiving the incentive signal $p_{\text {eq }}^{r}$, each PHEV agent will locally match $p_{\text {eq }}^{r}$ in its own demand vector, which amounts to the individual charging power ${ }^{i} P_{0}=\left\{{ }^{i} \mathbf{P}_{p^{r}}^{\mathrm{dem}} \mid p^{r}=p_{\mathrm{eq}}^{r}\right\}, \quad \forall i \in \mathcal{F}_{c, 0}$. PHEVs with a corner priority lower than $p_{\mathrm{eq}}^{r}$ will charge at most ${ }^{i} P^{\mathrm{min}}$, while PHEVs with a corner priority greater than ${ }^{i} p_{\mathrm{eq}}^{r}$ will start charging at a charging power between ${ }^{i} P^{\min }$ and ${ }^{i} P^{\max }$ [indicated as the active priorities in Fig. 4(b)].

\section{Iteration of the Three Steps}

The three steps are continuously repeated to adapt towards a dynamic, uncertain environment. In terms of DSM of PHEV, there can be many sources of uncertainty: unknown arrival
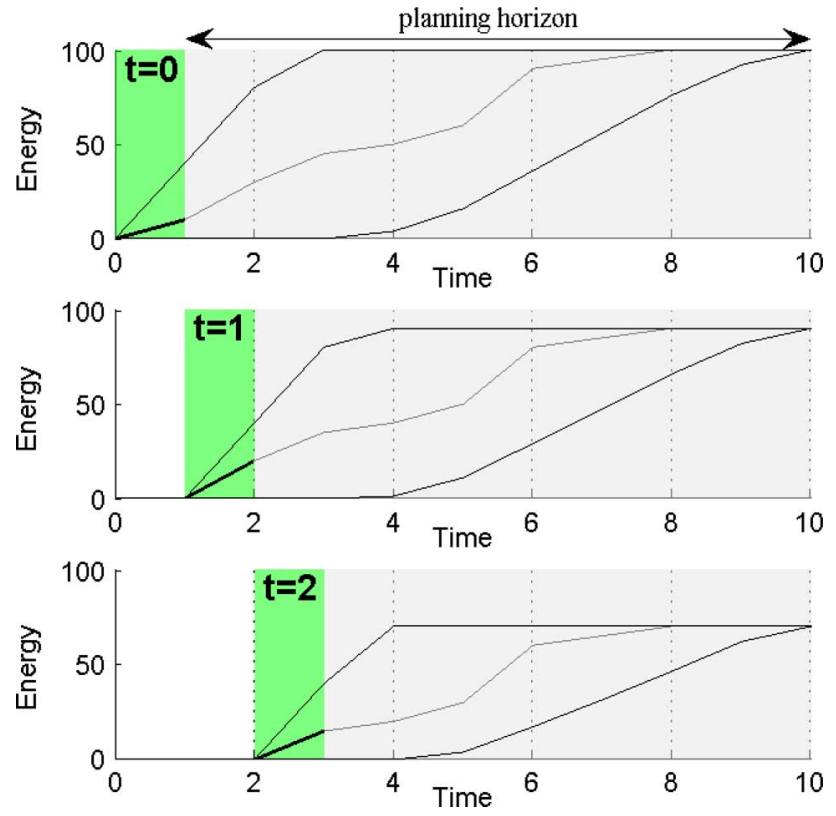

Fig. 5. Rolling horizon of the three-step approach.

times, stochastic departure times, inaccurate battery requirements, uncertain prices, etc. Through this iterative rolling horizon approach, energy and power constraints are continuously updated (step 1) to calculate a new charging plan (step 2) and control individual PHEVs (step 3). An example of the evolution of aggregated energy constraints, charging plan and online control action is shown in Fig. 5. In the evaluation, we consider unknown arrival times as source of uncertainty in the environment. As the evaluation is focussed on scalability, other sources of uncertainty are out of the scope of this paper.

\section{EVALUATION}

In this chapter, the proposed three-step approach is evaluated through comparison with a series of fully centralized benchmark solutions. These benchmark solutions can guarantee the best possible solution in terms of optimization objective, but are limited in terms of scalability. The goal of this evaluation is to determine to which degree our three-step approach can reach the best possible solution in terms of optimization objective, while improving scalability.

\section{A. Scenario}

The considered scenario is a residential PHEV fleet with two stakeholders:

1) Energy Supplier: wants to minimize the charging costs of its fleet. The costs made by charging PHEVs are described in an hourly-based cost model $C_{h}\left(E_{h}\right)$ in (20).

2) PHEV Owner: wants to charge its PHEV before departure. Consequently, PHEV owners provide their departure time when they arrive at home. The prediction of PHEV driving behavior is based on a statistical availability model [14]. This model represents the state of a car (home, driving, work, ...). To represent a realistic PHEV, the parameters from an Opel Ampera are chosen. To obtain a realistic initial SOC (state of charge), each simulation is ran one day beforehand starting with a full battery. This day is omitted from the simulation results. 


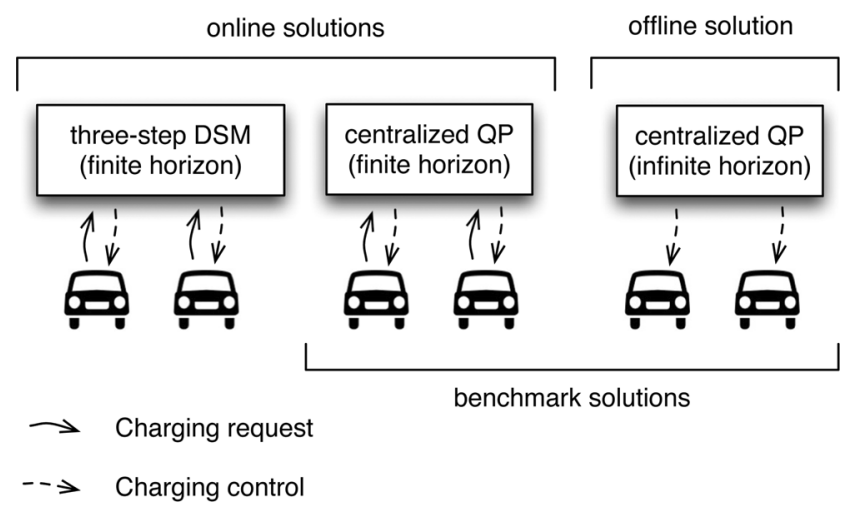

Fig. 6. Compared solutions.

\section{B. Energy Cost Model}

The energy cost model is based on the cost function $C_{h}\left(E_{h}\right)$ presented in [5], which is derived from a quadratic cost function for thermal generators [15]:

$$
C_{h}\left(E_{h}\right)=a_{h} E_{h}^{2}+b_{h} E_{h}+c_{h}
$$

where:

- $a_{h}$ is the wholesale price of electricity at each hour $h$.

- $E_{h}$ is the load of the whole fleet at each hour $h$.

- $b_{h}$ and $c_{h}$ are assumed 0 for simplicity.

This cost function can represent either the actual energy cost for thermal generators or simply artificial cost tariffs to impose proper load control [5]. The hourly wholesale prices are used from the Belgian power exchange platform Belpex [16] for the year 2010. The typical shape of the daily prices are low prices during the night and high prices during the evening (Fig. 8).

In step 2 of the three-step approach, this energy cost model is used as fleet objective function in (12). In the next section, the optimization problem for the benchmark solutions is described, which also uses this cost model as objective function.

\section{Benchmark Solutions}

To evaluate the three-step approach in the described scenario, two centralized benchmark solutions are defined (Fig. 6). Both these solutions are based on QP (quadratic programming) to optimize towards the quadratic cost function in (20).

The first benchmark is an online QP solution. Instead of aggregating PHEV constraints, the online solution uses individual PHEV constraints to schedule each grid-connected PHEV $i \in$ $\mathcal{F}_{c, t}$ its energy usage. To account for connecting PHEVs, this optimization is repeated every hour. The online QP problem is defined as

$$
\min _{{ }^{1} \mathbf{E}, \ldots,{ }^{n} \mathbf{E}} \sum_{h=1}^{t_{\text {end }}} a_{h}\left(\sum_{i=1}^{n}{ }^{i} E_{h}\right)^{2}
$$

subject to :

$$
\begin{array}{ll}
{ }^{i} E_{h}={ }^{i} E^{\max } & h \in\left\{{ }^{i} t_{\mathrm{dep}}\right\}, \\
{ }^{i} E_{h+1}={ }^{i} E_{h}+{ }^{i} P_{h} \Delta t & \forall h \in\left\{0, \ldots,{ }^{i} t_{\mathrm{dep}}\right\}, \\
0 \leq{ }^{i} P_{h} \leq{ }^{i} P^{\max } & \forall h \in\left\{0, \ldots,{ }^{i} t_{\mathrm{dep}}\right\},
\end{array}
$$

$$
\sum_{i=1}^{n}{ }^{i} P_{h} \leq P_{h}^{\text {limit }} \quad \forall h \in\left\{0, \ldots,{ }^{i} t_{\mathrm{dep}}\right\},
$$

$$
\forall i \in \mathcal{F}_{c, t} .
$$

with ${ }^{i} E^{\max }$ the maximum battery content of PHEV $i$. Accordingly, PHEVs fully charge their batteries, given unknown driving times.

The second benchmark is an offline QP solution, which creates one offline schedule based on the complete and exact charging and driving information of all PHEVs in the future.

$$
\min _{{ }^{1} \mathbf{E}, \ldots,{ }^{n} \mathbf{E}} \sum_{h=1}^{t_{\text {end }}} a_{h}\left(\sum_{i=1}^{n}{ }^{i} E_{h}\right)^{2}
$$

subject to :

$$
\begin{array}{ll}
0 \leq{ }^{i} E_{h} \leq{ }^{i} E^{\max } & \forall h \in\left\{0, \ldots, t_{\text {end }}\right\}, \\
{ }^{i} E_{h+1}={ }^{i} E_{h}+{ }^{i} P_{h} \Delta t & \forall h \in\left\{0, \ldots, t_{\text {end }}\right\}, \\
0 \leq{ }^{i} P_{h} \leq{ }^{i} P^{\max } & \forall h \in\left\{0, \ldots, t_{\text {end }}\right\}, \\
\sum_{i=1}^{n}{ }^{i} P_{h} \leq P_{h}^{\text {limit }} & \forall h \in\left\{0, \ldots, t_{\text {end }}\right\},
\end{array}
$$

$$
\begin{aligned}
& \forall i \in \mathcal{F}_{c, t}, \\
&{ }^{i} E_{h+1}={ }^{i} E_{h}-{ }^{i} D_{h} \Delta t \quad \forall h \in\left\{0, \ldots, t_{\text {end }}\right\}, \\
& \forall i \in \mathcal{F} \backslash \mathcal{F}_{c, t} .
\end{aligned}
$$

where ${ }^{i} D_{h}$ is battery energy required for driving PHEV $i$. Note that $t_{\text {end }}$ is the optimization horizon in the offline QP solution. In practical simulations, $t_{\mathrm{end}}$ is the final timepoint of the given data profiles.

\section{Simulation Results: Solution Quality}

In this part of the evaluation, the three-step approach is benchmarked in terms of total cost, as defined in (20). If no information is available about driving times of a PHEV (as in the three-step approach and online benchmark), PHEVs are charged as much as possible, given the power limitations of their household connection. If information about driving times is available (as in the offline benchmark), PHEVs are charged to maximize their electric driving time.

The total charging cost is assessed in a simulation of a scenario with 100 cars during 31 days. Each solution is simulated 100 times with different driving cycles for each car. In Fig. 7, the distributions of the total cost resulting from each solution are plotted in a histogram. On the right end of the histogram is uncontrolled charging, because most cars arrive in the evening and start charging during the highest prices. On the left end of the histogram is the offline benchmark, yielding the lowest charging cost due to an infinite optimization horizon.

The difference between the results of the offline and online benchmark solution is the "online gap." This gap is inherent to the online solution's limited optimization horizon, in contrary to the offline solution's infinite optimization horizon. The results of the offline benchmark solution show that, during weekdays, PHEVs are charged just enough to drive electrically. During weekends, however, PHEVs are fully charged to benefit from 


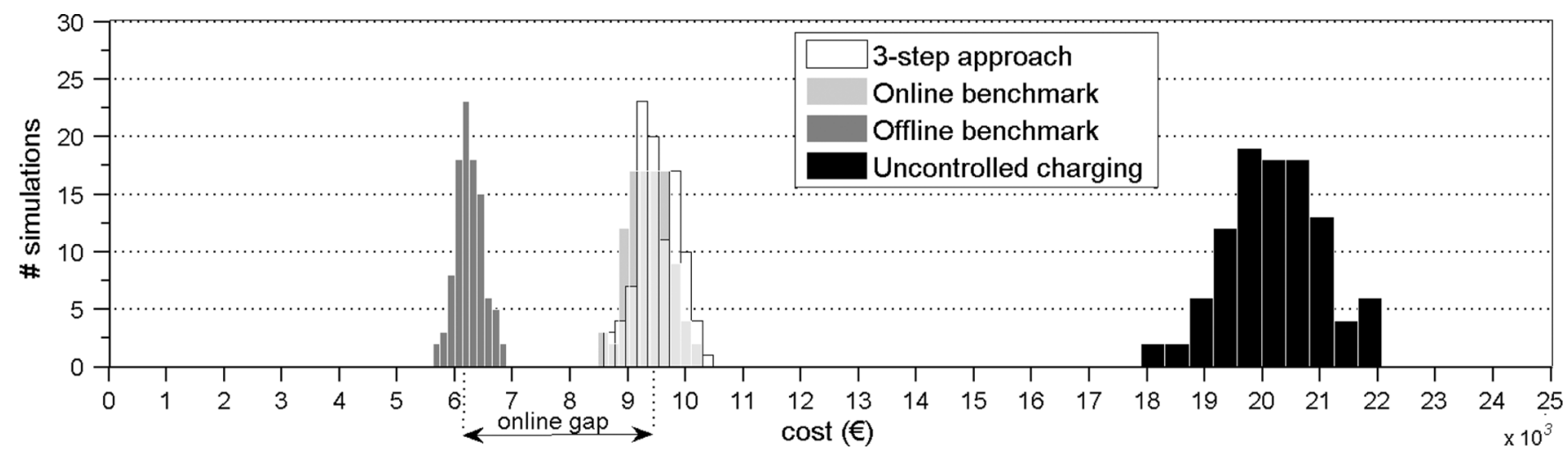

Fig. 7. Distribution of the total cost for each solution as result of a Monte-Carlo experiment.

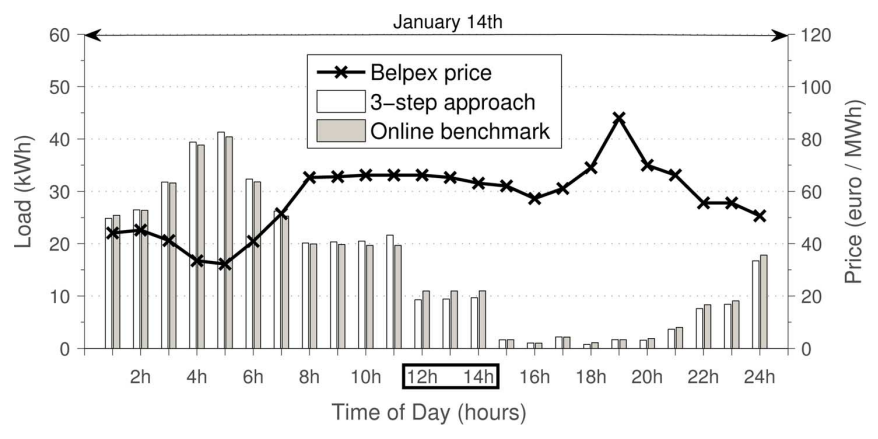

Fig. 8. Typical simulation run of a weekday (January 14th, 2010).

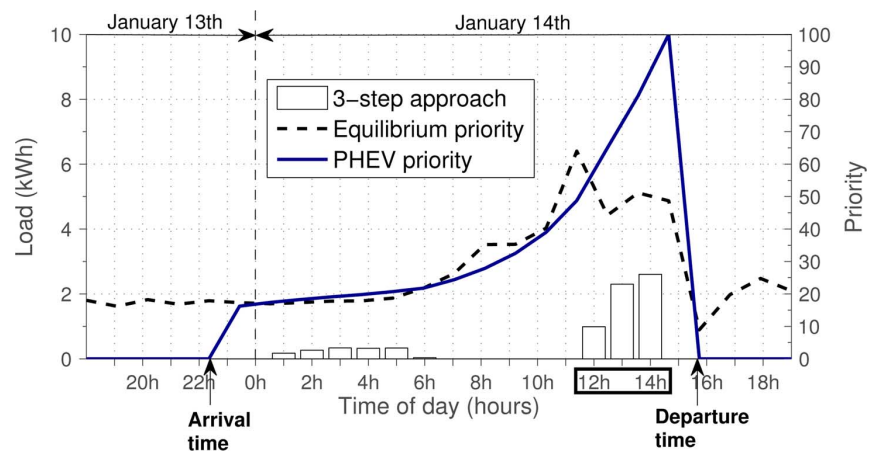

Fig. 9. Evolution of the global equilibrium priority and the local corner priority of one car.

the typically lower electricity prices. In a real setting, this solution is undesirable because PHEV owners do not exactly know their driving times beforehand.

The distribution of the total charging cost of the three-step approach and the online benchmark are similar (Fig. 7). While the offline benchmark has all information and uncontrolled charging none, the online benchmark has exactly the same amount of information as the three-step approach. The only difference is that the online benchmark individually schedules PHEVs, while the three-step approach uses aggregated constraints and a market-based incentive signal. On average, the online benchmark outperforms the three-step approach with $1.5 \%$ less total charging costs. This difference is further analyzed in a typical simulation run (Fig. 8).

In this simulation run, the online benchmark solution outperformed the three-step approach with $1.4 \%$. This difference is a direct result of a suboptimal division of $P_{0}$ between PHEVs (step 3, Section IV-C). In Fig. 9, the charging behavior of one car, managed by the three-step approach, is shown. During hour 1 to 5, the corner priority of this PHEV is a small fraction higher than the global equilibrium priority, causing this car to charge. Other cars, which leave earlier, should have been charged during this time, but are charged less.

While the priority scheme does not ensure a perfect division between PHEVs like the online benchmark, a close approximation is achieved by weighing between departure time, required energy and maximum charging power of PHEVs in the corner priority heuristic (18).

\section{E. Simulation Results: Scalability}

In this part of the evaluation, the scalability of the three-step approach is benchmarked by comparing its execution time with the online QP solution ${ }^{1}$. Because the PHEV scheduling problem can be defined as a convex optimization problem, execution time of the QP solution is known to be bounded by a polynomial. In the proceeding experiments, this polynomial is estimated and compared to the time complexity of the three-step approach. The input to a PHEV scheduling problem, which indicates the scale of the problem, is defined by the number of cars (vertical scalability) and the length of their optimization horizon (horizontal scalability), shown in Fig. 10.

1) Vertical Scalability (Fig. 11): To evaluate vertical scalability of the three-step approach, 10000 to 100000 cars are scheduled within a horizon of 144 time slots (e.g., one day for time slots of $10 \mathrm{~min}$ ). Each simulation is repeated 10 times with different PHEVs. Results show that the execution time of the three-step approach is constant, while the centralized solution scales as a 3rd order polynomial.

2) Horizontal Scalability (Fig. 12): To evaluate horizontal scalability of the three-step approach, 50000 cars are scheduled within a growing scheduling horizon from 10 to 140 time slots. Each simulation is repeated 10 times with different PHEVs. The results show that the execution time of the three-step approach scales linearly, while the centralized solution scales as a 5th order polynomial.

In summary, the proposed three-step approach ensures a constant execution time in terms of vertical scalability, due to an optimization independent of the PHEV fleet size (step 2,

\footnotetext{
${ }^{1}$ Simulations are performed using a workstation with Intel Xeon processor (3.46 GHz, $12 \mathrm{MB}$ cache, 4 cores) and $12 \mathrm{~GB}$ of ram.
} 


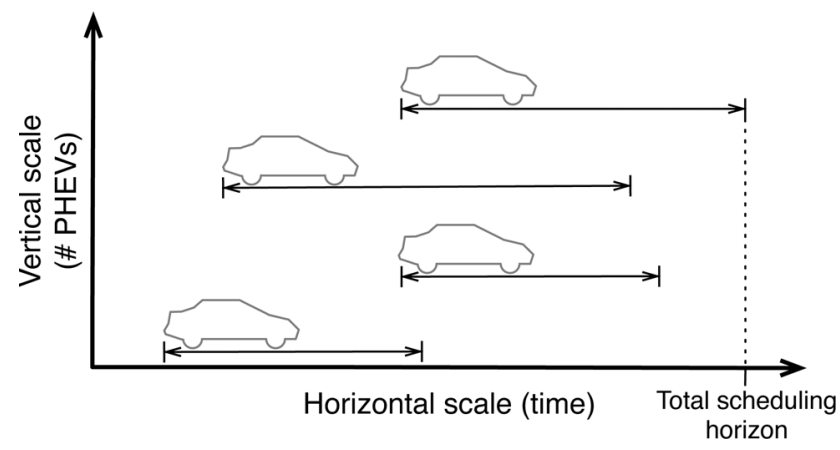

Fig. 10. Vertical and horizontal scalability in a PHEV scheduling problem.

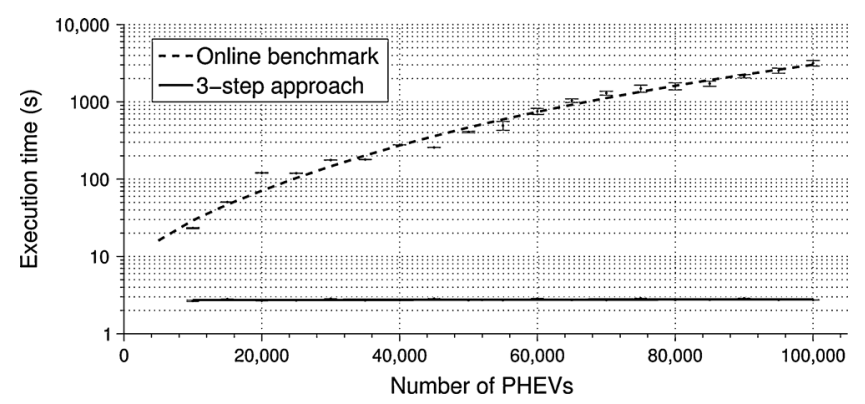

Fig. 11. Vertical scalability analysis.

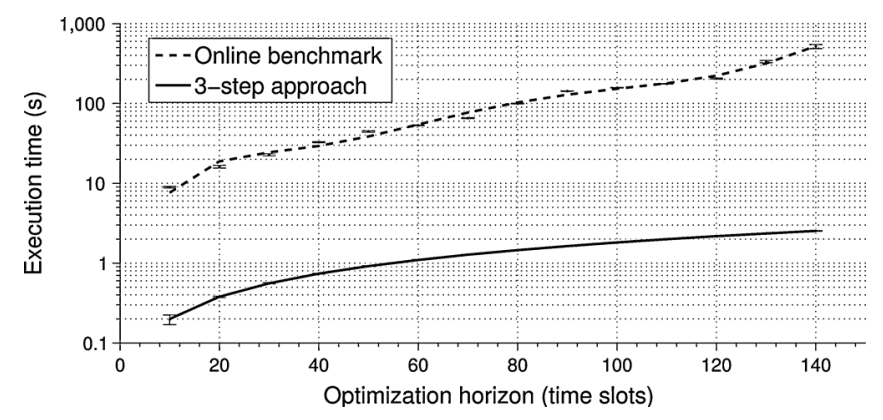

Fig. 12. Horizontal scalability analysis.

Section IV-B) and a linear execution time in terms of horizontal scalability. For both directions of scalability, the online benchmark calculates a solution several magnitudes slower, with polynomial time complexity.

\section{F. Simulation Results: Conclusion}

In this chapter, the proposed three-step approach was evaluated through comparison with a series of centralized benchmark solutions. Simulation results show that the offline benchmark calculates an optimal, but unrealistic result, while the online benchmark performs only $1.5 \%$ better than the three-step approach in terms of cost minimization. The cause for this difference is the suboptimal priority scheme used in step 3 of our approach. In terms of scalability, the execution time of QP problems is polynomial bounded, while the three-step approach scales linearly with the optimization horizon and remains constant with the amount of cars.

\section{General ConCLuSion}

In the future smart grid, DSM will be important to assure grid stability and secure power supply. To enable large-scale deployment of DSM, scalable DSM of local devices is essential.
Furthermore, an uncertain and dynamic environment has to be taken into account.

The solution presented in this article is a three-step, agentbased approach for DSM of PHEVs that combines the optimality of centralized DSM approaches with the scalability of decentralized DSM approaches. Essential in this three-step approach is the aggregation of local PHEV constraints through energy and power constraint vectors, which are used for scalable calculation of a charging plan for the PHEV fleet.

Simulations show that the three-step approach performs comparable to an online, centralized QP solution. An average cost difference of $1.5 \%$ between both solutions is caused by the difference between per-car optimization (QP solution) and a heuristic priority scheme (three-step approach). In exchange for this small difference, the three-step approach provides a realistic, decentralized solution with good scalability qualities. While the focus of this paper is scalability of execution time in a dynamic environment, communication scalability and privacy of local PHEV charging requirements are also expected to improve over centralized solutions.

\section{FUTURE WORK}

Current and future work focuses on the evaluation of the three-step approach in a variety of business models (e.g., deployment of PHEVs as a reserve capacity, balancing of unpredictable wind generation) and in an environment with a heterogenous group of consumers and generators. One aspect herein is the usage of stochastic optimization for integration of predictions (e.g., predictions of departure times, generation from renewables) Another aspect herein is the integration of grid constraints (e.g., power constraints of transformers and cables, limitation of voltage levels). Besides scalability of computations, communication scalability will also be a topic of future research. To assess communication scalability, we are developing an event-based three-step approach, where PHEVs asynchronously send their energy and power constraints towards the PHEV fleet agent.

\section{ACKNOWLEDGMENT}

This work is the result of a collaboration between the DistriNet research group of the Department of Computer Science (Catholic University of Leuven), VITO (the Flemish Institute for Technological Research) and the Electa research group of the Department of Electrical Engineering (Catholic University of Leuven).

\section{REFERENCES}

[1] "International energy agency," World Energy Outlook 2010 OECD-Organisation for Economic Cooperation and Development.

[2] "Lighting Research Program: Project 3.2. Energy efficient load-shedding lighting technology final report," California Energy Commission Public Interest Energy Research Program, Tech. Rep., Oct. 2005.

[3] PG\&E Corporation, 2012, Pacific Gas and Electric Co..

[4] A. Molderink, V. Bakker, M. G. C. Bosman, J. L. Hurink, and G. J. M. Smit, "Management and control of domestic smart grid technology," IEEE Trans. Smart Grid, vol. 1, no. 2, pp. 109-119, Sep. 2010.

[5] A. Mohsenian-Rad, V. W. S. Wong, J. Jatskevich, R. Schober, and A. Leon-Garcia, "Autonomous demand-side management based on gametheoretic energy consumption scheduling for the future smart grid," IEEE Trans. Smart Grid, vol. 1, no. 3, pp. 320-331, Dec. 2010. 
[6] M. D. Galus, R. L. Fauci, and G. Andersson, "Investigating phev wind balancing capabilities using heuristics and model predictive control," in Proc. 2010 IEEE Power Energy Soc. Gen. Meet., Jul. 2010, pp. 1-8.

[7] J. Nocedal and S. J. Wright, Numerical Optimization. New York: Springer, Aug. 2000.

[8] W. B. Powell, Approximate Dynamic Programming: Solving the Curses of Dimensionality, ser. Wiley Series in Probability and Statistics. Malden, MA: Wiley-Interscience, 2007.

[9] K. Clement, E. Haesen, and J. Driesen, "The impact of charging plug-in hybrid electric vehicles on a residential distribution grid," IEEE Trans. Power Syst., vol. 25, no. 1, pp. 371-380, Feb. 2010.

[10] R. N. Anderson, A. Boulanger, W. B. Powell, and W. Scott, "Adaptive stochastic control for the smart grid," Proc. IEEE, vol. 99, no. 6, pp. 1098-1115, Jun. 2011.

[11] J. Xu and V. W. S. Wong, "An approximate dynamic programming approach for coordinated charging control at vehicle-to-grid aggregator," in Proc. IEEE Int. Conf. Smart Grid Commun. (SmartGridComm), Oct. 2011, pp. 279-284.

[12] K. Kok, M. Scheepers, and R. Kamphuis, "Intelligence in electricity networks for embedding renewables and distributed generation," in Intelligent Infrastructures, ser. Intelligent Systems, Control and Automation: Science and Engineering Series. New York: Springer, 2009.

[13] S. Meisel, Anticipatory Optimization for Dynamic Decision Making, ser. Operations Research/Computer Science Interfaces Series. Dordrecht, The Netherlands: Springer, 2011.

[14] J. V. Roy, N. Leemput, S. D. Breucker, F. Geth, P. Tant, and J. Driesen, "An availability analysis and energy consumption model for a flemish fleet of electric vehicles," in Proc. Eur. Elec. Veh. Congr. (EEVC), Brussels, Belgium, Oct. 26-28, 2011.

[15] N. Ruiz, I. Cobelo, and J. Oyarzabal, "A direct load control model for virtual power plant management," IEEE Trans. Power Syst., vol. 24, no. 2, pp. 959-966, May 2009.

[16] Belpex. Belgian power exchange [Online]. Available: http://www. belpex.be/index.php?id=78 2011

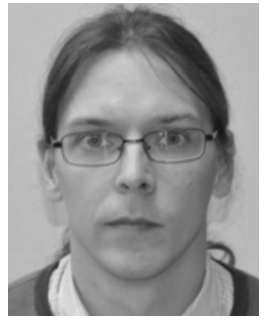

Bert Claessens was born in Neeroeteren, Belgium. He received his M.Sc. and Ph.D. degrees in applied physics from the University of Technology of Eindhoven, The Netherlands, in 2002 and 2006, respectively. In 2006 he started working at ASML Veldhoven, The Netherlands, as a design engineer. Since June 2010 he has been working as a Researcher at the Vlaamse Instelling voor Technologisch Onderzoek (VITO), Mol, Belgium. His research interests include algorithm development and data analysis.

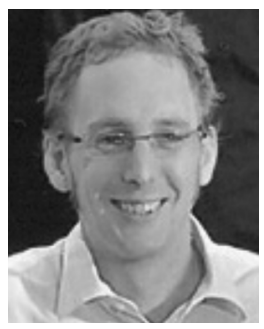

Maarten Hommelberg was born in Tilburg on October 9,1978 . He graduated in the field of building services in 2005. His graduation subject was titled "Software Agents for a Building Management System." His first employer was ECN (Energy Research Centre of The Netherlands). There, he was Researcher and Project Leader of several projects among the Virtual Power Plant based on CHP. In March 2008, he started working at VITO, Mol. Belgium, where he is Project Manager in the field of smart grids.

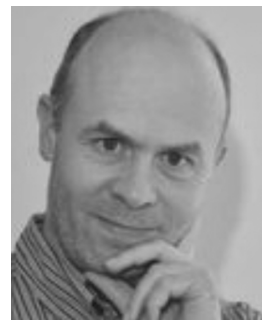

Tom Holvoet received his Ph.D. degree in computer science from the Katholieke Universiteit Leuven, Belgium. He is a Professor at the Department of Computer Science, Katholieke Universiteit Leuven. His research interests include software engineering of decentralized and multi-agent systems, software architecture, autonomic computing, and aspect-oriented software development. Contact him at DistriNet, Dept. of Computer Science, KU Leuven, Celestijnenlaan 200A, B-3001 Leuven, Belgium; tom.holvoet@cs.kuleuven.be.

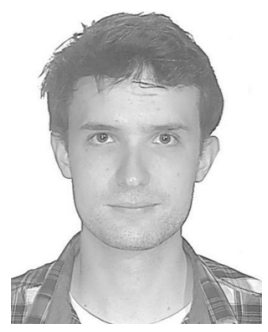

Stijn Vandael was born in Hasselt, Belgium. He received the M.S. degree in industrial engineering from the Katholieke Hogeschool Limburg (KHLim), Belgium, and the M.Sc. degree from the Katholieke Universiteit Leuven (KU Leuven), Belgium. Currently, he is working towards a Ph.D. degree at the department of Computer Science (KU Leuven) in close cooperation with the department of Electrical Engineering (KU Leuven). His research interests include coordination in multi-agent systems, plug-in hybrid electric vehicles, and smart grids.

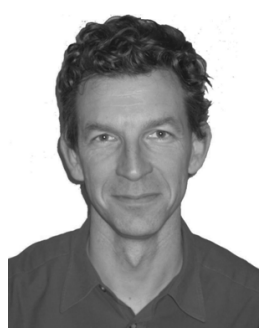

Geert Deconinck received the M.Sc. degree in electrical engineering and the Ph.D. degree in applied sciences from the Katholieke Universiteit Leuven (KU Leuven), Leuven, Belgium, in 1991 and 1996, respectively. He is a professor at the Department of Electrical Engineering, Katholieke Universiteit Leuven. His research interests include the design, analysis, and assessment of software-based fault-tolerant solutions to meet real-time, dependability, and cost constraints for embedded applications on parallel and distributed systems. Contact him at Electa, Dept. of Electrical Engineering, KU Leuven, Kasteelpark Arenberg 10, B-3001 Leuven, Belgium; geert.deconinck@esat.kuleuven.be. 